spiel waren wir 2019 Mitsponsor eines großen Treffens zur Differentialgeometrie bei MATRIX, einer Forschungsstation in Australien, in der Nähe von Melbourne. Solche Aktivitäten können von unserem Koordinatorprojekt unterstützt werden. In dieser Hinsicht ist es sehr flexibel.

Wie ist es für Sie, das SPP zu leiten? Gibt es viel Verwaltungsarbeit, viel Bürokratie?

Mit dem SPP konnte ich eine Teilzeitassistentin einstellen, Alexandra Linde, und sie hilft mir sehr bei der Verwaltung. Dazu gehört die Organisation der Evaluationskolloquien und die Abwicklung der Kostenerstattung an Personen, die Koordinatormittel für Konferenzen verwenden. All das wäre ohne die wertvolle Hilfe von Alexandra nicht zu schaffen.

Ein weiterer Teil der Verwaltungsarbeit, der eher zur wissenschaftlichen Seite gehört und ziemlich spannend war, war das Einrichten der SPP-Website. Das ist wirklich ein wesentlicher Teil der Infrastruktur. Für mich war es das erste Mal, dass ich so etwas gemacht habe.

Es klingt vielleicht ein bisschen seltsam, aber ich genieße die Arbeit. Es ist wie eine große Party. Ich bin in Kontakt mit Forschungsgruppen gekommen, die ich vorher nicht so wahrgenommen habe, und mir ist klar ge- worden, dass sie wirklich gute Arbeit leisten. Mein Blick auf die Forschung in Deutschland und auf die Geometrie im Allgemeinen hat sich sehr erweitert. Das begrüße ich sehr.

Wir vom Programmkomitee sind diejenigen, die das Geld verteilen. Wir machen also die Leute glücklich! Auf die konkrete Forschung haben wir keinen Einfluss, denn die diesbezüglichen Entscheidungen werden innerhalb der einzelnen Forschungsprojekte getroffen. Wir haben einige der Highlights bei den Veranstaltungen organisiert, zum Beispiel die SPP-Konferenz, und das war fantastisch, weil wir wussten, dass unser Budget es uns erlaubt, eine Konferenz auf höchstem Niveau zu organisieren.

Dinge wie das Einrichten der Webseite, das Organisieren des Kickoff-Meetings oder auch dieses Interview, das wir gerade führen, liegen außerhalb der üblichen wissenschaftlichen Arbeit, und das macht mir Spaß. Für mich ist Wissenschaft mehr, als sich nur auf das eigene Forschungsprojekt zu konzentrieren. Wir haben eine gewisse Verantwortung, anderen zu vermitteln, was wir tun, und das spielt auch im SPP eine Rolle. Ich persönlich mag das sehr. Die Arbeit im SPP ist eine perfekte Kombination von Verwaltungsarbeit und wissenschaftlichen Ergebnissen, ein ideales Zusammenspiel von beidem.

Bernhard Hanke (Promotion 1999) studierte Mathematik und theoretische Physik an der LMU München, der University of Cambridge und der Universität Bonn. Längere Forschungsaufenthalte verbrachte er an der University of Notre Dame, an der Universität Göttingen, an der TU Berlin, am IMPA (Rio de Janeiro), am IHES (Bures-sur-Yvette) sowie am Courant Institute of Mathematical Sciences (New York). Nach einer Professur an der TU München ist er seit 2010 Inhaber des Lehrstuhls für Differentialgeometrie an der Universität Augsburg.

\title{
Geometrisch definierte asymptotische Koordinaten in der Allgemeinen Relativitätstheorie
}

\author{
Carla Cederbaum
}

\begin{abstract}
Gemeinsam mit Jan Metzger (Universität Potsdam) leite ich im Rahmen des SPP „Geometrie im Unendlichen“ ein Projekt an der Grenze zwischen Geometrischer Analysis und Mathematischer Relativitätstheorie. In diesem Projekt beschäftigen wir uns mit der Geometrie des asymptotischen Bereichs von „relativistischen Anfangsdaten“, also wortwörtlich mit deren Geometrie im Unendlichen. Genauer befassen wir uns mit verschiedenen Blätterungen des asymptotischen Bereichs und mit dazugehörigen Erhaltungsgrößen sowie deren Koordinaten(un-)abhängigkeit.
\end{abstract}

(Relativistische) Anfangsdaten $(M, g, K)$ bestehen aus einer Riemannschen Mannigfaltigkeit $(M, g)$, hier dreidimensional, sowie einem symmetrischen $(0,2)$-Tensorfeld $K$ auf $M$. Wie der Name nahelegt, beschreiben solche Anfangsdaten den „Anfangszustand“ $M=\{t=0\}$ einer Raumzeit, also ei- ner zeit-orientierten Lorentzschen Mannigfaltigkeit; $g$ ist dann die von der Raumzeitmetrik induzierte Riemannsche Metrik, das Tensorfeld $K$ ist die zweite Fundamentalform (oder „extrinsische Krümmung“) der betrachteten Hyperfläche $M=\{t=0\}$ bezüglich deren zukunftsgerichteter Nor- 
male. Besonders interessieren wir uns in diesem Projekt für Anfangsdaten, die isolierte Systeme beschreiben. Mathematisch modelliert wird dies durch die Annahme, die betrachteten Anfangsdaten $(M, g, K)$ mögen asymptotisch flach sein: Außerhalb eines kompakten Inneren $\mathcal{K}$ sollen asymptotische Koordinaten

$$
\vec{x}: M \backslash \mathcal{K} \rightarrow \mathbb{R}^{3} \backslash \overline{B_{1}(\overrightarrow{0})}
$$

existieren; die Komponenten von $g$ und $K$ sollen in diesen Koordinaten für $r:=|\vec{x}| \rightarrow \infty$ und festes $k \in \mathbb{N}_{\geq 2}, \varepsilon>0$ abfallen wie

$$
\begin{aligned}
g_{i j} & =\delta_{i j}+\mathcal{O}_{k}\left(r^{-\frac{1}{2}-\varepsilon}\right), \\
K_{i j} & =\mathcal{O}_{k-1}\left(r^{-\frac{3}{2}-\varepsilon}\right),
\end{aligned}
$$

wobei $\delta=\left(\delta_{i j}\right)$ die Euklidische Metrik bezeichnet, siehe auch Abb. 1. Zusätzlich sollen die Energiedichte $\mu \in C^{\infty}(M)$ und die Komponenten der Impulsdichte $J \in \Gamma\left(T^{*} M\right)$ für $r \rightarrow \infty$ abfallen wie

$$
\begin{aligned}
16 \pi \mu & :=\mathrm{R}_{g}-|K|_{g}^{2}-\left(\operatorname{tr}_{g} K\right)^{2}=\mathcal{O}_{k-2}\left(r^{-3-2 \varepsilon}\right), \\
-8 \pi J_{i}: & =\operatorname{div}_{g}\left(K-\left(\operatorname{tr}_{g} K\right) g\right)_{i}=\mathcal{O}_{k-2}\left(r^{-3-2 \varepsilon}\right),
\end{aligned}
$$

wobei $\mathrm{R}_{g}$ die Skalarkrümmung von $(M, g)$ bezeichnet. Insbesondere sind damit die $L^{1}$-Volumenintegrale von $\mu$ und $J$ über das (asymptotische) Ende $M \backslash \mathcal{K}$ endlich. Der Index in der $\mathcal{O}$-Notation zeigt dabei an, wie viele Koordinatenableitungen ebenfalls als entsprechend schnell abfallend angenommen werden, z. B. müssen die $i$-ten partiellen Ableitungen einer Funktion $f=\mathcal{O}_{1}\left(r^{s}\right), s \in \mathbb{R}, \partial_{i} f=\mathcal{O}\left(r^{s-1}\right)$ erfüllen. Typischerweise verwenden wir gewichtete Sobolevräume anstelle der durch die $\mathcal{O}$-Notation nahegelegten (gewichteten) $C^{k}$-Räume.

Die geometrischen Invarianten Energie, Impuls und Masse

Die Gesamtenergie $E=E(M, g, K) \in \mathbb{R}$ und der Gesamtimpuls $\vec{P}=\vec{P}(M, g, K) \in \mathbb{R}^{3}$ von asymptotisch flachen Anfangsdaten $(M, g, K)$ wurden von Arnowitt, Deser und Misner durch koordinatenabhängige Formeln definiert, die auf einen Hamiltonschen-Systeme-Zugang zurückgehen. Aus Arbeiten von Bartnik bzw. Chruściel wissen wir, dass diese Formeln tatsächlich geometrische Invarianten der Anfangsdaten, also nicht koordinatenabhängig sind. Für schwächere Abfallbedingungen gilt dies nicht; die Bedingungen (1), (2) sind also in dieser Hinsicht optimal.

Das berühmte von Schoen und Yau bzw. Witten bewiesene Positive Masse-Theorem besagt nun, dass geodätisch vollständige, asymptotisch flache Anfangsdaten, die der physikalischen Bedingung $\mu \geq|J|_{g}$ genügen, automatisch auch $E \geq|\vec{P}|_{\delta}$ erfüllen. Gleichheit gilt genau dann, wenn $(M, g, K)$ Anfangsdaten für die Minkowski-Raumzeit der Speziellen Relativitätstheorie sind und damit insbesondere $E=0, \vec{P}=\overrightarrow{0}$. Somit ist die Gesamtmasse $m=m(M, g, K) \in \mathbb{R}$,

$$
m:=\sqrt{E^{2}-|\vec{P}|_{\delta}^{2}}
$$

wohldefiniert und positiv (außer im Minkowski-Fall, dort gilt $m=0$ ). Die Formel (3) entspricht genau der Formel für die Ruhemasse $m$ eines Punktteilchens in der Speziellen Relativitätstheorie, aufgeteilt in die Energie $E$ und den Impuls $\vec{P}$, den das Teilchen aus der Sicht eines frei fallenden Beobachters hat. Ruht das Teilchen relativ zum Beobachter $(\vec{P}=\overrightarrow{0})$, erhalten wir $m=E$, besser bekannt als $E=m c^{2}$, wobei $c=1$ für die Lichtgeschwindigkeit steht, die hier durch Wahl der Einheiten eins gesetzt ist.

Geometrische und analytische Fragen zum Schwerpunkt

Während Masse, Energie und Impuls von asymptotisch flachen Anfangsdaten also sehr gut verstandene geometrische Invarianten sind, sind andere zentrale physikalische Größen wie etwa der Schwerpunkt solcher Anfangsdaten analytisch und geometrisch noch nicht vollständig verstanden. Zwar gibt es in der mathematischen und physikalischen Literatur eine breit anerkannte koordinatenabhängige Formel für den Gesamtschwerpunkt $\vec{z}_{\mathrm{RTBO}^{\prime}}=\vec{z}_{\mathrm{RTBO}^{\prime}}(M, g, K) \in \mathbb{R}^{3}$ von asymptotisch flachen Anfangsdaten $(M, g, K)$, ähnlich denen für Energie und Impuls. Sie wurde von Regge-Teitelboim und Beig-O'Murchadha aus einem Hamiltonschen-SystemeZugang abgeleitet. Allerdings ist bekannt, dass $\vec{z}_{\mathrm{RTBO}}$ für viele Anfangsdaten bzw. für viele asymptotische Koordinatensysteme auf gegebenen Anfangsdaten divergiert. Bisher behilft man sich durch von Regge-Teitelboim beschriebene zusätzliche asymptotische Symmetriebedingungen in Koordinaten, die die Konvergenz von $\vec{z}_{\mathrm{RTBO}}$ sicherstellen. Diese Regge-Teitelboim-Bedingungen sind jedoch bisher geometrisch nicht gut verstanden. Ein Ziel unseres Forschungsprojektes ist, sie geometrisch besser zu verstehen oder, falls notwendig, durch geeignete geometrische Bedingungen in Form von geometrischen, partiellen Differentialgleichungen an die Koordinaten zu ersetzen.

Ein weiteres Problem des Schwerpunkts $\vec{z}_{\mathrm{RTBO}}$ ist, dass er sich nicht zwingend äquivariant unter der asymptotischen Poincarégruppe der umgebenden Raumzeit transformiert. Anders ausgedrückt ändert sich der durch die Formel berechnete Schwerpunkt unter (asymptotischem) Beobachterwechsel nicht wie erwartet wie der beobachtete Aufenthaltsort eines Punktteilchens in der Speziellen Relativitätstheorie. Gemeinsam mit Anna Sakovich habe ich vor kurzem eine Poincaré-äquivariant korrigierte Schwerpunktsdefinition $\vec{z}_{\text {äquiv. }}$ hergeleitet. Die erwähnten Divergenzprobleme bleiben auch für $\vec{z}_{\text {äquiv. }}=\vec{z}_{\mathrm{RTBO}}+$ Korrektur bestehen; Konvergenz kann wiederum durch Regge-TeitelboimBedingungen sichergestellt werden. Somit ist die geplante Untersuchung geometrischer Bedingungen an die Koordinaten auch für diese Schwerpunktsdefinition relevant. Gleichzeitig stellt sich die Frage, unter welchen Umständen bzw. in welchen Koordinaten die vorgenommene Korrektur (nicht) verschwindet; dem werden wir in unserem Projekt ebenfalls nachgehen.

Ein gänzlich anderer, sehr eleganter Zugang zur Definition des Schwerpunkts asymptotisch flacher Anfangsdaten wurde von Huisken und Yau gewählt. Unter der An- 


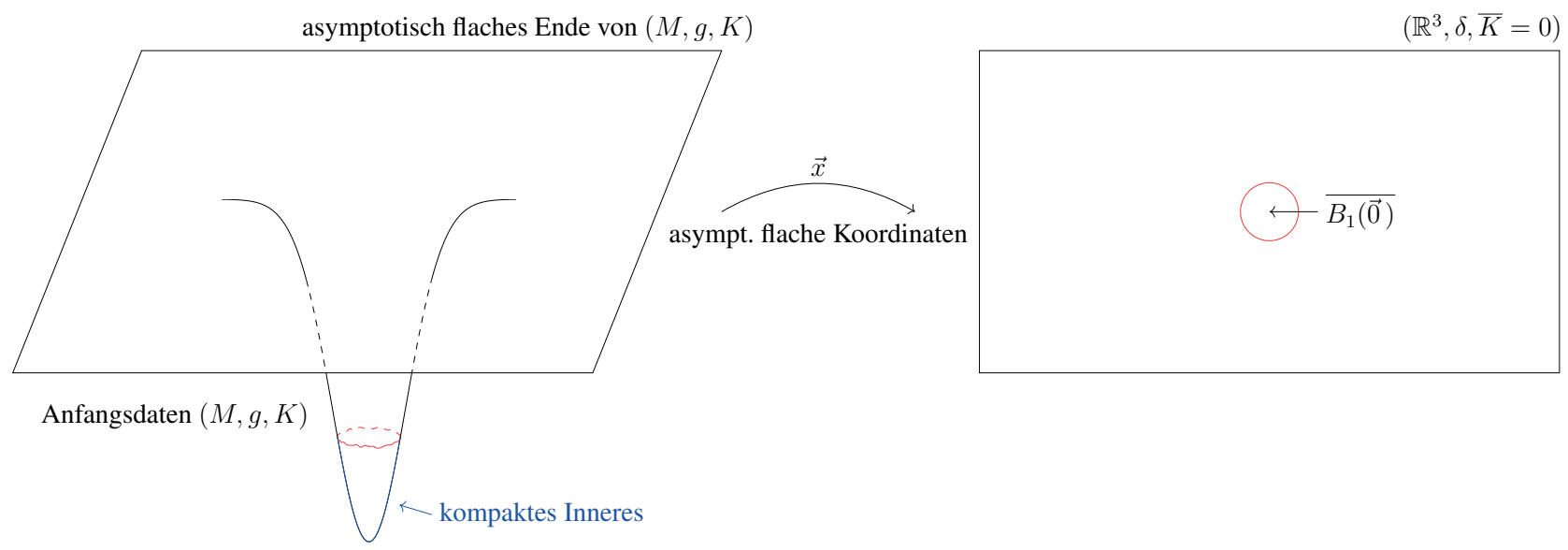

Abbildung 1. Anfangsdaten $(M, g, K)$ - aufgeteilt in ein kompaktes Inneres und ein asymptotisch flaches Ende - sowie die auf diesem Ende definierten asymptotischen Koordinaten $\vec{x}: \mathbb{R}^{3} \backslash \overrightarrow{B_{1}(\overrightarrow{0})}$.

nahme asymptotischer Rotationssymmetrie konnten sie zeigen, dass das asymptotisch flache Ende von Anfangsdaten $(M, g, K)$ mit positiver Energie $E>0$ auf eindeutige Weise durch zweidimensionale Flächen $\Sigma_{H}$ konstanter mittlerer Krümmung $H$ (engl. constant mean curvature, CMC) geblättert ist, siehe Abb. 2. Zusätzlich erhalten sie sehr präzise Abschätzungen, die zeigen, dass die CMC-Blätter $\Sigma_{H}$ asymptotisch für $H \searrow 0$ sehr schnell intrinsisch und extrinsisch rund werden. Der CMC-Schwerpunkt $\vec{z}_{\text {CMC }}$ der Anfangsdaten bzgl. der asymptotischen Koordinaten $\vec{x}$ ist dann definiert als

$$
\vec{z}_{\mathrm{CMC}}:=\lim _{H \searrow 0} \frac{1}{\left|\Sigma_{H}\right|_{\delta}} \int_{\Sigma_{H}} \vec{x} d A_{\delta}
$$

also als Grenzwert der Euklidischen Mittelpunkte der Flächen $\vec{x}\left(\Sigma_{H}\right) \subset \mathbb{R}^{3}$, siehe Abb. 2. Diese weit über die Relativitätstheorie hinaus sehr einflussreiche Arbeit wurde inzwischen von Metzger, Huang, Nerz etc. auf optimale asymptotische flache Anfangsdaten wie in (1), (2) mit $E \neq 0$ verfeinert. Zusätzlich wurde dabei gezeigt, dass $\vec{z}_{\mathrm{CMC}}=\vec{z}_{\mathrm{RTBO}}$ gilt; somit leidet auch dieser Schwerpunktsbegriff unter Konvergenzproblemen, nachgewiesen gemeinsam mit Christopher Nerz, und unter fehlender asymptotischer PoincaréÄquivarianz.

Gemeinsam mit Anna Sakovich habe ich vor kurzem eine neue asymptotische Blätterung für optimal asymptotisch flache Anfangsdaten wie in (1), (2) mit $E \neq 0$ vorgeschlagen und deren Existenz und Eindeutigkeit sowie asymptotische Rundheitsabschätzungen bewiesen. Diese STCMCBlätterung besteht aus zweidimensionalen Flächen $\Sigma_{\mathcal{H}}$ konstanter mittlerer Raumzeitkrümmung $\mathcal{H}:=|\overrightarrow{\mathcal{H}}|_{\text {Raumzeit }}$ (engl. constant spacetime mean curvature, STCMC), d. h. die Lorentzsche Länge des Kodimension 2 mittleren Krümmungsvektors $\overrightarrow{\mathcal{H}}$ der Fläche $\Sigma_{\mathcal{H}}$ in der umgebenden Raumzeit ist konstant. Sie lässt sich aus den Anfangsdaten berechnen als

$$
\mathcal{H}=\sqrt{H^{2}-\left(\operatorname{tr}_{\Sigma} K\right)^{2}}=\text { const. }
$$

Die STCMC-Blätterung ist an die asymptotische PoincaréInvarianz der umgebenden Raumzeit angepasst; entsprechend transformiert sich der entsprechend (4) definierte STCMC-Schwerpunkt $\vec{z}_{\text {STCMC }}$ automatisch Poincaréäquivariant, also wie der Aufenthaltsort eines Punktteilchens in der Speziellen Relativitätstheorie. Es gilt $\vec{z}_{\text {STCMC }}=$ $\vec{z}_{\text {äquiv. }}$; auch $\vec{z}_{\text {STCMC }}$ leidet unter Konvergenzproblemen, die wir in diesem Projekt besser verstehen und durch Konstruktion geeigneter Koordinaten lösen wollen. Dazu wollen wir die geometrische Form der CMC-Blätter mit denen der STCMC-Blätterung und von verwandten Blätterungen vergleichen und daraus präzise geometrische und analytische Einsichten über das asymptotische Ende relativistischer Anfangsdaten herleiten.

\section{Einbindung ins Schwerpunktprogramm SPP 2026 Geometry at Infinity}

Anlässlich des gemeinsam organisierten und aus SPPKoordinatorenmitteln finanziell unterstützten Minisymposiums Mathematical Perspectives on General Relativity bei der DMV-Tagung 2019 in Karlsruhe kamen Jan Metzger und ich ins Gespräch über einen größeren gemeinsamen Projektantrag im SPP 2026. Zuvor hatten wir zwar immer wieder gerne gemeinsam über Mathematik diskutiert und auch bereits eine Frühjahrsschule für junge Wissenschaftlerinnen und Wissenschaftler organisiert, hatten aber bisher kein konkretes gemeinsames Projekt verfolgt.

Die Ausschreibung der im SPP-Projekt bewilligten Postdoc-Stelle hat unser Projekt nach außen sichtbar gemacht und zu in diesem Maße unerwarteter Vernetzung 

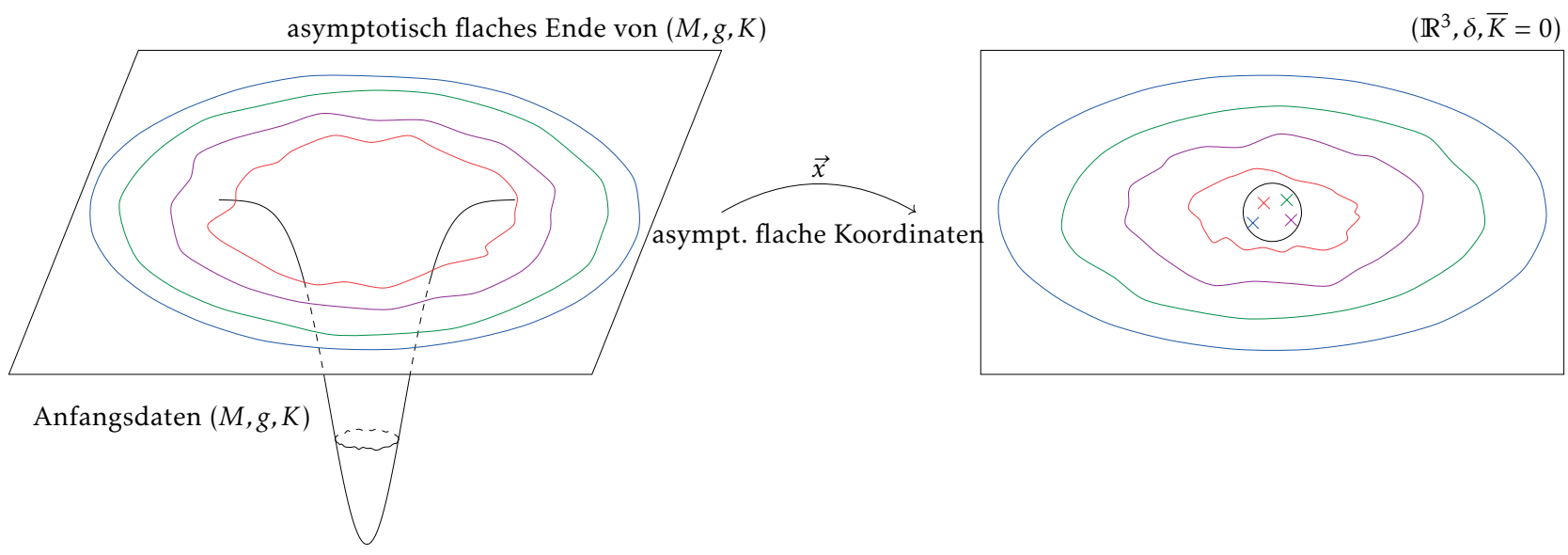

Abbildung 2. Asymptotisch flache Anfangsdaten $(M, g, K)$ mit einzelnen (ST)CMC-Blättern $\Sigma$ (in Farbe) sowie deren Bilder $\vec{x}(\Sigma)$ und (Euklidische) Mittelpunkte (farbige Kreuze).

mit jungen Wissenschaftlerinnen und Wissenschaftlern aus dem Ausland geführt. Wir werden die uns durch die Projektförderung und ggf. die SPP-Koordinatorenmittel zur Verfügung stehenden Mittel für Reisen und Gäste sicherlich nach Ende der Reisebeschränkungen auch sehr gut nutzen können, um diese neu entstanden wissenschaftlichen Verbindungen auszubauen.

Bereits die gemeinsame Arbeit am Antrag war wissenschaftlich sehr stimulierend und hat uns geholfen, eine tragfähige Kooperation aufzubauen. Obwohl unsere Projektförderung noch nicht begonnen hat, treffen sich unsere beiden Arbeitsgruppen und die zukünftige Postdoktorandin Dr. Melanie Graf (zur Zeit University of Washington, Seattle) seit einer Weile jeden Monat zu einem Onlineseminar, bei dem wir Fragen und Ergebnisse rund um die Themen des SPP-Projekts besprechen und wichtige Vorarbeiten gemeinsam lesen. Dadurch strahlt das Projekt auf unsere Arbeitsgruppen aus und wirkt durch den gezielten Austausch gerade in den isolierenden Corona-Zeiten sehr stimulierend.

Wir planen, während der Laufzeit des SPP 2026 eine weitere Frühjahrsschule für junge Wissenschaftlerinnen und Wissenschaftler auszurichten und hoffen, dass diese für viele Mitglieder des SPP und darüber hinaus von Interesse sein wird.

Ich bin sehr gespannt auf das Kennenlernen der anderen SPP-Projekte und der darin tätigen Wissenschaftlerinnen und Wissenschaftler und auf daraus eventuell entstehende Folgeprojekte.

Prof. Dr. Carla Cederbaum, Universität Tübingen, Fachbereich Mathematik, Auf der Morgenstelle 10, 72076 Tübingen cederbaum@math.uni-tuebingen.de

Carla Cederbaum ist Professorin für Differentialgeometrie und Mathematische Relativitätstheorie an der Eberhard Karls Universität Tübingen, seit 2019 ist sie Mitglied im hier vorgestellten SPP 2026 "Geometrie im Unendlichen“. 2016 erhielt sie den Manfred Fuchs Preis der Heidelberger Akademie der Wissenschaften und ist seitdem Kollegiatin der Akademie. Sie engagiert sich seit vielen Jahren in der Mathematik-Kommunikation und wurde dafür unter anderem zweimal zur DMV-Mathemacherin des Monats ernannt. 